\title{
Searching for emission line and OB stars in Cl 1806-20 using a NIR narrow-band technique
}

Michelle L. Edwards ${ }^{1,2}$, Reba M. Bandyopadhyay ${ }^{2}$, Stephen S. Eikenberry $^{2}$, Valerie J. Mikles ${ }^{2,3}$ and Dae-Sik Moon ${ }^{4}$

${ }^{1}$ Gemini Observatory, Southern Operations Center, La Serena, Chile email: medwards@gemini.edu

${ }^{2}$ Department of Astronomy, University of Florida, Gainesville, FL 32611

${ }^{3}$ Department of Physics and Astronomy, Louisiana State University, Baton Rouge, LA 70803

${ }^{4}$ Department of Astronomy and Astrophysics, University of Toronto, Toronto M5S3H8, Canada

\begin{abstract}
We survey the environment of $\mathrm{Cl}$ 1806-20 using near-infrared narrow-band imaging to search for $\mathrm{Br} \gamma$ features indicative of evolved massive stars. Using this technique, we successfully detect previously identified massive stars in the cluster. We detect no new emission line stars, establishing a firm upper limit on the number of Wolf Rayets and Luminous Blue Variables; however, we do find several candidate OB supergiants, which likely represent the bulk of the heretofore undiscovered massive star population.
\end{abstract}

Keywords. stars: emission-line, open clusters and associations: general

\section{Introduction}

Discovered by Fuchs et al. (1999), Cl 1806-20 is home to a variety of interesting and rare objects, including a candidate Luminous Blue Variable (LBV 1806-20), multiple Wolf Rayets (WRs), a soft-gamma repeater (SGR 1806-20), and several OB supergiants (Fuchs et al. 1999, Eikenberry et al. 2004, Figer et al. 2005). Although individual members of Cl 1806-20 have been identified on a case-by-case basis with spectroscopy, no systematic effort to census the cluster's massive stellar population exists in the literature. To better constrain the membership of $\mathrm{Cl}$ 1806-20 we performed near-infrared narrow- and broad-band imaging to search for massive candidate cluster members. We focused on $\operatorname{Br} \gamma$ emission lines indicative of stellar winds in massive stars (Hanson et al. 1996, Figer et al. 1997, Blum et al. 2001) and Br $\gamma$ absorption found in OB supergiants (Hanson et al. 1996).

\section{Observation and Analysis}

On 2005 August 26-27, we used the Wide Field Infrared Camera (WIRC) (Wilson et al. 2003 ) on the Palomar 200" telescope to obtain $J, K_{s}, 2.16 \mu \mathrm{m} \mathrm{Br} \gamma$, and $2.27 \mu \mathrm{m} K_{\text {cont }}$ images of an 8.7 arcminute $\times 8.7$ arcminute region around $\mathrm{Cl} 1806-20$. We reduced the data with FATBOY, a PYTHON based data pipeline developed at the University of Florida, and performed astrometry on these images using KOORDS in the KARMA software package. We then completed PSF photometry on our science frames with DAOPHOT II and ALLSTAR (Stetson 1987, Stetson 1992). We calibrated the $J$ and $K_{s}$ magnitudes for our sources with 2-MASS photometry. Using TOPCAT, the Tool for OPeration on Catalogues and Table we matched data across all four bands. 


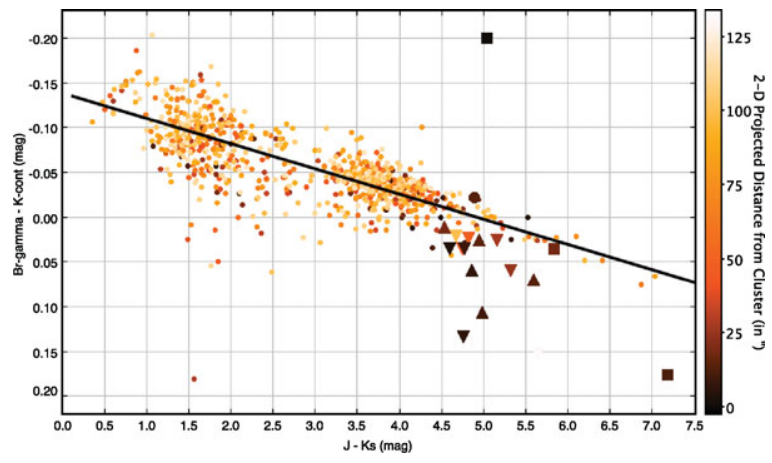

Figure 1. Color-color diagram of stars within an $4 \mathrm{pc}(2 \mathrm{arcmin})$ radius of $\mathrm{Cl} 1806-20$. OB supergiants are marked as triangles, WR stars as squares and the LBV as a circle. New OB supergiant candidates are marked as inverted triangles. The solid black line is the narrow-band zeropoint.

Using the resulting 4-band catalogue of sources we created a $J-K_{s}$ versus $B r \gamma-K_{\text {cont }}$ diagram (Fig. 1) of objects within a 2 arcmin radius from the cluster. We calculated the 2-D projected distance between each star and a point close to the center of the cluster.

Using an $A_{V}=29 \pm 2$ for $\mathrm{Cl} 1806-20$, (Corbel \& Eikenberry 2004) we find $A_{K}=$ $3.25 \pm 0.56$ and $A_{J}=8.18 \pm 0.22$ yielding a $J-K_{s}=4.93 \pm 0.34 \mathrm{mag}$ for the color of the cluster. We focused our search for cluster members in this region of the diagram.

\section{Results}

We confirmed the existence of known massive stars in Cl 1806-20. Several of our reported equivalent widths are in good agreement with the literature values. Where discrepancies exist, we explored the reasons. We found that in some cases, insufficient information in the literature prevented quantitative comparison. In other cases, we found literature data or completed follow-up observations that indicated the discrepancy may be a result of intrinsic variations.

We did not detect any previously unknown WR or LBV stars in Cl 1806-20. This finding allows us to place a firm upper limit on the number of very massive stars in the cluster. However, we did find a population of candidate OB supergiants that may represent the bulk of the heretofore undiscovered cluster population. We suggest that these stars should be targeted for future spectroscopic observations.

\section{References}

Blum, R. D., Schaerer, D., Pasquali, A., Heydari-Malayeri, M. et al. 2001, AJ, 122, 1875

Corbel, S. \& Eikenberry, S. S. 2004, A\& A, 419, 191

Eikenberry, S. S., Matthews, K., LaVine, J. L., Garske, M. A. et al. 2004, ApJ, 616, 506

Figer, D. F., McLean, I. S., \& Najarro, F. 1997, ApJ, 486, 420

Figer, D. F., Najarro, F., Geballe, T. R., Blum, R. D. et al. 2005, ApJ (Letters) 622, L49

Fuchs, Y., Mirabel, F., Chaty, S., Claret, A. et al. 1999, A\&SA, 350, 891

Hanson, M. M., Conti, P. S., \& Rieke, M. J. 1996, ApJS, 107, 281

Stetson, P. B. 1987, PASP, 99, 191

Stetson, P. B. 1992, in: D. M. Worrall, C. Biemesderfer, \& J. Barnes (eds.), Astronomical Data Analysis Software and Systems I, ASP-CS 25, p. 297

Wilson, J. C., Eikenberry, S. S., Henderson, C. P., Hayward, T. L. et al. 2003, in: M. Iye \& A. F. M. Moorwood (eds.), Instrument Design and Performance for Optical/Infrared Ground-based Telescopes, SPIE Conference Series 4841, p. 451 